\title{
Two-level cervical arthroplasty using a "no-distraction" technique
}

\author{
Deshpande Rajakumar, MCh, Ankit Sharma, DNB, Akshay Hari, DNB, Subhas Konar, MCh, \\ and Murali Krishna, $\mathrm{MCh}$
}

Department of Neurosurgery, Fortis Hospitals, Bangalore, India

Cervical arthroplasty is being recognized as an emerging alternative to anterior cervical fusion with comparable or superior outcomes. The authors describe the surgical nuances of 2-level cervical arthroplasty in a case of 2-level degenerative disease. In this surgical technique, conventional vertebral body distraction has been avoided to prevent facet distraction, which can be a cause of persistent postoperative neck pain. Good motion preservation was observed at the 1-year follow-up examination.

The video can be found here: https://youtu.be/YTpRVRXuZZk.

KEYWORDS 2-level cervical arthroplasty; motion preservation; no-distraction technique; facet distraction prevention; video 